\title{
Brain MRI Findings in Neurologically Asymptomatic Patients with Infective Endocarditis
}

A. Hess, I. Klein, B. lung, P. Lavallée, E. Ilic-Habensus, Q. Dornic, F. Arnoult, L. Mimoun, M. Wolff, X. Duval, and J.-P. Laissy

\section{ABSTRACT}

BACKGROUND AND PURPOSE: Neurologic complications in infective endocarditis are frequent and affect patient prognosis negatively. Additionally, detection of asymptomatic lesions by MR imaging could help early management of this condition. The objective of our study was to describe MR imaging characteristics of cerebral lesions in a neurologically asymptomatic population with infective endocarditis.

MATERIALS AND METHODS: One hundred nine patients at the acute phase of a definite or possible infective endocarditis according to the Duke modified criteria and without neurologic manifestations according to the NIHSS were prospectively included. Each patient underwent cerebral MR imaging and MRA within 7 days of admission.

RESULTS: MR imaging showed abnormalities in 78 patients (71.5\%). Acute ischemic lesions (40 patients, 37\%) and cerebral microbleeds (62 patients, 57\%) were the most frequent lesions. Eight patients had an acute SAH, 3 patients had brain microabscesses, 3 had a small cortical hemorrhage, and 3 had a mycotic aneurysm. Acute ischemic lesions mostly appeared as multiple small infarcts disseminated in watershed territories $(25 / 40,62.5 \%)$ and as lesions of different ages (21/40, 52.5\%). Cerebral microbleeds were preferentially distributed in cortical areas (362/539 cerebral microbleeds, 67\%). No significant correlation was found among lesions, in particular between acute ischemia and cerebral microbleeds.

CONCLUSIONS: Occult cerebral lesions, in particular cerebral microbleeds and acute ischemic lesions, are frequent in infective endocarditis. The MR imaging pattern of acute small infarcts of different ages predominating in watershed territories and cortical cerebral microbleeds may represent a surrogate imaging marker of infective endocarditis.

ABBREVIATIONS: CMB = cerebral microbleed; IE = infective endocarditis; IMAGE-Endocarditis = Resonance Magnetic Imaging at the Acute Phase of Endocarditis

I nfective endocarditis is associated with symptomatic neurologic complications in $20 \%-40 \%$ of cases. ${ }^{1-4}$ Among symptomatic complications, ischemic stroke is the most common manifestation, whereas hemorrhagic stroke, brain abscess, cerebral hemorrhage or $\mathrm{SAH}$, and mycotic aneurysms are less frequent. ${ }^{2}$ Symptomatic cerebral complications are one of the main prognostic factors in infective endocarditis (IE) $)^{5-8}$ because they are significantly associated with a

Received September 23, 2012; accepted after revision October 26.

From the Service de Radiologie (A.H., I.K., J.-P.L.), Service de Cardiologie (B.I., L.M.), Service de Neurologie (P.L.), Service des Explorations Fonctionnelles (F.A.), and Service de Réanimation médicale et des maladies infectieuses (M.W.), EA 3964, Hôpital Universitaire Bichat, Paris, France; Institut National de la Santé et de la Recherche Médicale (Inserm) U698 (I.K., P.L., J.-P.L.), Paris, France; Université Paris Diderot (B.I., P.L., M.W., X.D., J.-P.L.), Paris 7, UFR de Médecine, site Bichat, Paris, France; Inserm CIC 007 (E.I.-H., X.D.), AP-HP, Hôpital Universitaire Bichat, Paris, France; Inserm U738 (Q.D.), Paris, France; and Inserm U738 (X.D.), Paris, France.

Please address correspondence to Agathe Hess, MD, Hôpital Universitaire Bichat, 46 rue Henri Huchard, 75748 Paris Cedex, France; e-mail: agathehess@gmail.com

- Indicates open access to non-subscribers at www.ajnr.org

indicates article with supplemental on-line tables

http://dx.doi.org/10.3174/ajnr.A3582 3-month mortality rate. ${ }^{2,3,9,10}$ Symptomatic neurologic complications are difficult to predict and prevent because they mostly occur in the early phase of IE and have been reported as the presenting symptom of the disease in up to $47 \%$ of cases. $^{2}$

Asymptomatic cerebral lesions revealed by systematic cerebral CT and/or MR imaging have been recently reported, but their impact on patient care and prognosis has not been completely assessed..$^{8,11-13}$ Our group showed that detection of cerebral asymptomatic lesions influenced diagnostic decisions in cases of suspected IE. ${ }^{14,15}$

The purposes of the present study were to extensively describe MR imaging characteristics of cerebral lesions in a neurologically asymptomatic population at the acute phase of IE, to prospectively report their respective frequencies, and to assess correlations between different MR imaging features.

\section{MATERIALS AND METHODS \\ Patients}

One hundred fifty consecutive patients included in the previously published IMAGE-Endocarditis study ${ }^{15}$ prospectively underwent 
brain MR imaging for clinically suspected IE according to the Duke modified criteria (On-line Tables 1 and 2$)^{16}$ between June 2005 and September 2008.

Among them, 41 patients were excluded because MR imaging was not interpretable or not hardware-archived $(n=15)$, because the diagnosis of IE was finally excluded $(n=10)$, or because they had acute neurologic symptoms related to IE at the clinical examination performed before MR imaging ( $n=16$, NIHSS $>0$ ). Consequently, the population study consisted of 109 patients with definite $(n=84)$ or possible $(n=25)$ IE. The local ethics committee approved the study. All patients gave written informed consent.

\section{Brain Imaging Protocol}

MR imaging was performed on a single Signa HDx 1.5T system (GE Healthcare, Chalfont St. Giles, UK) within 7 days after inclusion in the IMAGE study. We used a standardized protocol, including T1WI, DWI, FLAIR, and T2* sequences.

DWI was acquired with a single-shot EPI spin-echo sequence with TR/TE, 8000/87.6 ms; FOV, $26 \times 26 \mathrm{~cm}^{2}$; matrix, $128 \times 128$; NEX, two; 24 sections; 5 -mm section thickness; and $0.3-\mathrm{mm}$ section gap. The trace images were calculated from 3 diffusionweighted acquisitions with gradients sequentially applied along each of the 3 orthogonal axes $\left(b=1000 \mathrm{~s} / \mathrm{mm}^{2}\right)$. The acquisition parameters of FLAIR were as follows: TR/TE/TI, 8002/158.2/2000 ms; matrix, $320 \times 192$; NEX, 1; with all other parameters being identical. For the $\mathrm{T} 2{ }^{\star}$ sequence, the parameters were the following: TR/TE, 750/17.3 ms; flip angle, 20; FOV, $24 \times 19.5 \mathrm{~cm}^{2}$; matrix, $416 \times 224$; NEX, one; 5 -mm section thickness; $0.5-\mathrm{mm}$ section gap.

After intravenous injection of contrast medium (gadoterate meglumine, Dotarem; Guerbet, Alnauy-sous-Bois, France), we performed an elliptic centric MRA (TR/TE, 8.6/3 ms; FOV, $24 \times$ $18 \mathrm{~cm}^{2}$; matrix, $512 \times 416$; NEX, 0.75; 0.8-mm section thickness; -0.4-mm section gap) and a $3 \mathrm{D}$ T1 sequence (TR/TE, 13.1/5.3 ms; FOV, $25.6 \times 20.5 \mathrm{~cm}^{2}$; matrix, $256 \times 256$; NEX, 0.5; $1.2-\mathrm{mm}$ section thickness; $-0.6-\mathrm{mm}$ section gap).

\section{MR Imaging Analysis}

A standardized form, first tested on an independent sample to collect relevant information, was used to interpret the MR imaging. The 60 initial MR images were reviewed by 2 readers (A.H. and I.K.), with, respectively, 3 and 10 years of experience in neuroradiology, blinded to each other and to patient characteristics. In case of a discrepancy, diagnosis was obtained by consensus. After satisfactory concordance was reached, the remaining MR images $(n=49)$ were assessed by 1 reader (A.H.).

Acute ischemic lesions were classified as territorial infarcts and small cortical or subcortical lesions. We additionally defined 3 topographic patterns of ischemic lesions: 1) a single lesion including either a territorial infarct, a small cortical lesion, or a small subcortical lesion; 2) multiple lesions associating a territorial infarct and 1 or several small cortical and/or subcortical lesions; and 3) multiple small cortical and/or subcortical infarcts distributed in the watershed territories. The infarct age was defined by using an ADC map, FLAIR, and postcontrast 3D T1 sequences as either "recent," including acute ischemia ( $<8$ days) with low ADC and subacute ischemia ( $<3$ months) with normalized ADC and con- trast enhancement, or "chronic" (>3 months) with high ADC and no contrast enhancement.

Cerebral microbleeds were defined as round $\mathrm{T} 2{ }^{\star}$ hypointensities with a diameter $\leq 10 \mathrm{~mm}$. We collected their number, size ( $\leq 5$ or $5-10-\mathrm{mm}$ ), localization (cortical including meningeal location, subcortical, or posterior fossa), and signal (homogeneous or heterogeneous).

SAH was defined as linear FLAIR hyperintensities or T2* hypointensities following the shape of cortical sulci. We collected their localization (adjacent to a cerebral microbleed [CMB], acute ischemic or hemorrhagic lesion, isolated), number (single or multiple), and age (acute or chronic).

Parenchymal hemorrhage was detected by $\mathrm{T}^{*}$ and FLAIR sequences. Additionally, a T1 sequence was used to evaluate the age of bleeding.

Cerebral abscess was defined as an expansive lesion with central restricted $\mathrm{ADC}$, peripheral edema, annular enhancement \pm $\mathrm{T} 1$ hyperintensity, and $\mathrm{T} 2{ }^{*}$ hypointensity of the abscess capsule. We collected the number (single or multiple), size, and adjacent abnormalities (ischemic lesion or CMB).

Mycotic aneurysms were searched by using a postcontrast 3D T1 sequence and MRA. They were defined by using angiographic criteria described elsewhere. ${ }^{17,18}$ Briefly, each aneurysm had to be either distal (ie, involving segments 2, 3, or 4 of the middle or posterior cerebral artery) or proximal in association with at least 2 other criteria: change in size or morphology on consecutive angiographies, presence of another intra- or extracranial mycotic aneurysm, rupture of the aneurysm, arterial occlusion or stenosis adjacent to the aneurysm, or cerebral infarction at the level of the aneurysm. ${ }^{17}$ We added a fusiform shape in angiographic features as a diagnostic criterion. ${ }^{18}$ We also collected their number (single or multiple), size, arterial localization, and adjacent abnormalities (ischemic lesion, $\mathrm{CMB}, \mathrm{SAH}$, or parenchymal hemorrhage).

Leukoaraiosis was graded with the modified score of Fazekas et al. ${ }^{19}$

\section{In-Hospital Outcome}

Neurologic examination was performed during the follow-up in the patients who underwent cardiac surgery, to rule out the occurrence of symptomatic neurologic complications.

\section{Statistical Analysis}

Interobserver agreement was evaluated by using a simple $\kappa$ coefficient (for binary variables) and a weighted $\kappa$ coefficient (for ordinal variables).

We described MR imaging data as proportional and effective for qualitative variables and as mean and SD for continuous variables.

We used a $\chi^{2}$ test (or Fisher exact test for a sample size of $<5$ ) to assess associations between cerebral lesions. Statistical testing was done at the 2-tailed $\alpha$ level of .05. Data were analyzed with the SAS package, Release 9.3 (SAS institute, Cary, North Carolina).

\section{RESULTS}

\section{Patients}

Clinical characteristics of patients are summarized in Table 1. The mean age was 59 years, $76 \%$ were men, and oral streptococci $(25 \%)$ and Staphylococcus aureus (24\%) accounted for half of the cases. 
Table 1: Clinical characteristics of 109 neurologically asymptomatic patients with $\mathrm{IE}^{\mathrm{a}}$

\section{Characteristic}

\begin{tabular}{lc}
\hline Age (yr) (mean \pm SD) (range) & $59 \pm 15.4,24-87$ \\
Men & $79(72.5 \%)$ \\
Definite IE & $84(77 \%)$ \\
Possible IE & $25(23 \%)$ \\
Previous history of IE & $13(12 \%)$ \\
Microorganism & \\
Oral streptococci & $27(25 \%)$ \\
Group D streptococci & $8(7 \%)$ \\
Enterococci & $8(7 \%)$ \\
Staphylococcus aureus & $26(24 \%)$ \\
Coagulase-negative staphylococci & $5(4.5 \%)$ \\
Other microorganism or $>1$ microorganism & $14(13 \%)$ \\
No microorganism identified & $21(19 \%)$ \\
Prosthetic valve IE & $32(29 \%)$ \\
IE on native valve & \\
Aortic & $17(15.5 \%)$ \\
Mitral & $39(36 \%)$ \\
Aortic and mitral & $10(9 \%)$ \\
Tricuspid & $7(6.5 \%)$ \\
Other & $4(4 \%)$ \\
Prior anticoagulant or antiplatelet agent therapy & $36(33 \%)$ \\
\hline All datarepresented as numbers (percentages) except for age. & \\
\hline
\end{tabular}

a All data are presented as numbers (percentages) except for age.

${ }^{\mathrm{b}}$ According to Duke modified criteria.

Table 2: Interobserver agreement for MRI reading

\begin{tabular}{|c|c|}
\hline Cerebral Lesion & $\kappa$ Coefficient $(95 \% \mathrm{CI})$ \\
\hline Acute ischemic lesion & $0.89(0.74-0.90)$ \\
\hline \multicolumn{2}{|l|}{ CMBs } \\
\hline Located in cortical areas & $0.91(0.82-0.99)$ \\
\hline Located in subcortical areas & 0.70 (0.42 to 0.98$)$ \\
\hline$\leq 5 \mathrm{~mm}$ & $0.92(0.84-0.99)$ \\
\hline$>5 \mathrm{~mm}$ & $0.79(0.56-1.00)$ \\
\hline No. of CMBs (none, $1-3,>3$ ) ${ }^{a}$ & $0.94(0.89-0.99)$ \\
\hline Parenchymal hemorrhage and SAH & $0.72(0.41-1.00)$ \\
\hline Old ischemic lesions & $0.84(0.56-1.00)$ \\
\hline Leukoaraiosis score & $0.83(0.75-0.91)$ \\
\hline
\end{tabular}

Note:- $\mathrm{Cl}$ indicates confidence interval.

a Weighted $\kappa$ coefficient of $0.94(95 \% \mathrm{Cl}, 0.89-0.99)$ after categorization of the number of $\mathrm{CMBs}$ into 3 groups (none, 1-3, >3).

\section{Brain MR Imaging}

Interobserver Agreement. $\kappa$ coefficients for interobserver agreement were good regardless of the lesion (Table 2).

\section{Prevalence of MR Imaging Lesions}

MR imaging showed abnormalities in 78 patients ( $71.5 \%)$, and 32 patients (29\%) had several acute lesions. Acute ischemic lesions were found in 40 patients (37\%); CMBs, in 62 (57\%); SAH, in 8 (7\%); small cortical hemorrhage, in $3(3 \%)$; cerebral microabscess, in $3(3 \%)$; and mycotic aneurysm, in $3(3 \%)$. Twenty-five patients $(23 \%)$ had both acute ischemic lesions and CMBs.

\section{Description of MR Imaging Lesions}

Acute Ischemic Lesions. Acute ischemic lesions were observed in 40 patients (37\%) (Fig 1).

Most patients had the "watershed ischemia" pattern (pattern 3 ) with multiple acute small lesions distributed in junctional cortical and subcortical arterial territories $(n=25 / 40$; $62.5 \%)$, while 8 patients $(8 / 40 ; 20 \%)$ had a single acute ischemic lesion (pattern 1$)$ classified as a single territorial $(n=3)$, single small cortical $(n=3)$, or single small subcortical $(n=2)$ lesion. The last 7 patients $(17.5 \%)$ had pattern 2 (territorial and small cortical and/or subcortical infarcts). All 40 patients presented with supratentorial lesions, and 16 patients (16/40; $40 \%$ ) additionally had ischemic lesions located in the posterior fossa.

Ischemic lesions of different ages attested to by concomitant analysis of DWI, the ADC map, FLAIR, and postcontrast T1 imaging were observed in 21 patients $(21 / 40 ; 52.5 \%)$. Subacute ischemic lesions were found in 5 patients with pattern 2 $(5 / 21 ; 24 \%)$ and in 16 patients with pattern $3(16 / 21 ; 76 \%)$ (Fig 2).

\section{CMBs}

Sixty-two patients (57\%) had CMBs. Of these patients, $85.5 \%$ had CMBs located in cortical areas $(n=53)$ (Fig 3). Less frequently, CMBs were located in the subcortical white matter and basal ganglia (29 patients, $47 \%$ ) or in the posterior fossa (27 patients, $43.5 \%$ ).

Forty-six patients $(46 / 62 ; 74 \%)$ had multiple CMBs, and 33 patients $(53 \%)$ had $>3$ CMBs. Five hundred thirty-nine CMBs were found, with a mean of $8.7 \pm 19.26 \mathrm{CMBs}$ per patient. Among them, 362 CMBs (67\%) were located in cortical areas; 56 (10.5\%), in the subcortical white matter and basal ganglia; and 121 $(22.5 \%)$, in the posterior fossa. Sixty-five CMBs (12\% of all $\mathrm{CMBs}$ ) had a heterogeneous signal on $\mathrm{T}^{*}$ sequences. Thirty patients $(48.5 \%)$ presented with at least $1 \mathrm{CMB}$ with a heterogeneous signal. Twenty-one CMBs ( $4 \%$ of all CMBs) were greater than $5 \mathrm{~mm}$, and 13 patients (20\%) had at least $1 \mathrm{CMB}$ of $>5 \mathrm{~mm}$. Twenty of the 21 CMBs larger than $5 \mathrm{~mm}$ (95\%) were heterogeneous in signal.

CMBs and ischemic lesions were located in different areas. No $\mathrm{CMB}$ was found in watershed territories. Furthermore, no statistical association was found between $\mathrm{CMBs}$ and acute ischemic lesions $(P=.61)$, whatever the pattern $(P=.68$ for pattern $1, P=$ .11 for pattern 2 , and $P=.08$ for pattern 3 ). CMBs were not associated with older age (mean age, $58.2 \pm 2$ for patients with CMBs versus $60.1 \pm 2.2$ for patients without CMBs; $P=.5$ ).

\section{Acute SAH}

Small SAHs were found in 8 patients (7\%), adjacent to a mycotic aneurysm in 1 . Six patients with SAH (75\%) had CMBs, but no statistical association was found $(P=.17)$.

\section{Small Cortical Parenchymal Hemorrhage}

Small cortical parenchymal hemorrhages were found in 3 patients (3\%). Two were heterogeneous in signal, appearing hyper- and isointense in $\mathrm{T} 1$ and hyper- and hypointense in T2 sequences, consistent with subacute hemorrhage. One of the cortical hemorrhages occurred in a patient with a contralateral mycotic aneurysm. The third patient had a cortical hemorrhagic transformation of subacute ischemia.

\section{Brain Microabscesses}

Three patients (3\%) had brain microabscesses that were associated with multiple ischemic lesions and multiple CMBs in 2 patients. One patient had a mycotic aneurysm adjacent to the microabcesses. In 1 patient, microabscesses were also associated with 




FIG 1. Acute ischemic lesions on DWI in 3 different patients. A, Pattern 1: single territorial infarct. $B$, Pattern 2: territorial and small cortical and/or subcortical infarcts. C, Pattern 3: small ischemia in the watershed territories.
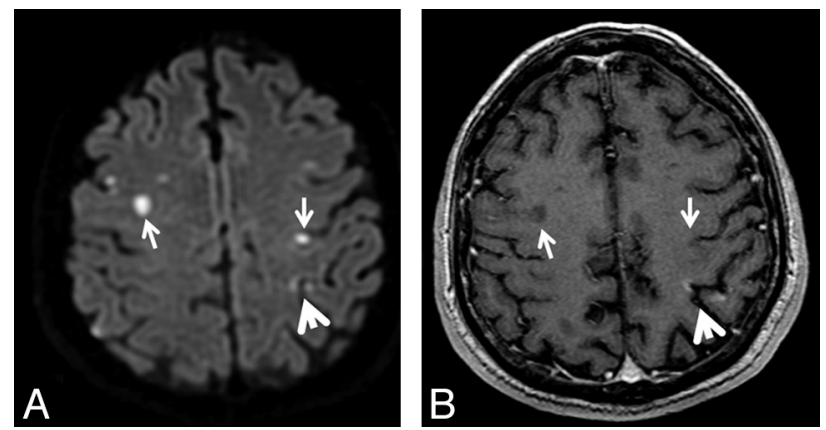

FIG 2. DWI $(A)$ and postcontrast $T 1(B)$ sequences show recent ischemic lesions of different ages in the same patient: acute ischemia (small white arrows) and subacute ischemia (large white arrows).
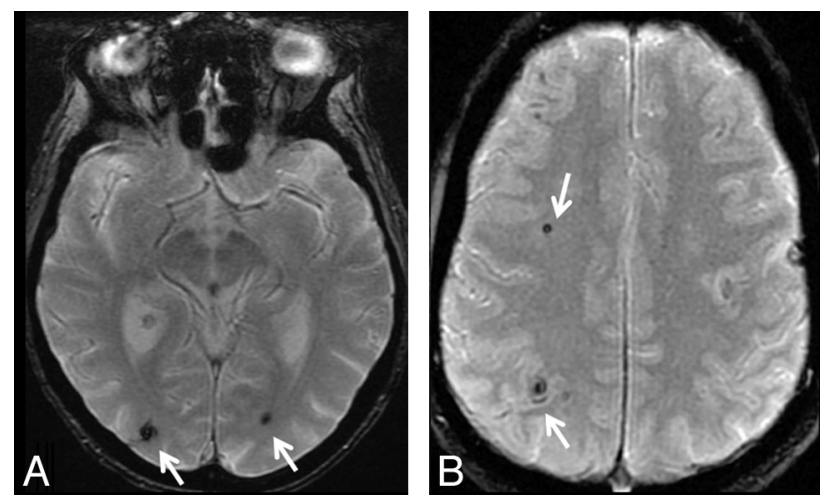

FIG 3. Multiple cortical and subcortical microbleeds on a $\mathrm{T}_{2}^{*}$ sequence.

adjacent leptomeningeal enhancement on postcontrast T1 sequences, consistent with meningitis.

\section{Mycotic Aneurysm}

Three mycotic aneurysms were found in 3 patients (3\%), all in distal branches of the middle cerebral artery M3 $(n=1)$ or M4 $(n=2)$, confirmed by conventional angiography in 2 cases. They were sacciform $(n=2)$ or fusiform $(n=1)$. These small aneurysms $(1.8,3$, and $3.4 \mathrm{~mm}$ in the widest diameter [mean, $2.4 \pm 0.9 \mathrm{~mm}$ ]) were detected by postcontrast $\mathrm{T} 1$ sequences and postcontrast MRA.

For 2 patients, the mycotic aneurysms also appeared as round susceptibility magnetic artifacts in $\mathrm{T}^{*}$ sequences and DWI. One patient had an acute ischemic lesion systematized to the left su-

\section{In-Hospital Outcome}

\section{DISCUSSION}

perficial sylvian territory near the mycotic aneurysm, and 1 patient had both acute $\mathrm{SAH}$ and microabscesses adjacent to the mycotic aneurysm. In the third patient, the mycotic aneurysm was distant from a contralateral small cortical hemorrhage.

\section{Old Ischemic Lesions and Leukoaraiosis}

Twenty-nine patients had old ischemic lesions $(26.5 \%)$, and 42 patients $(38.5 \%)$ had leukoaraiosis (punctuate, 24 [57\%]; early confluent, 15 [36\%]; confluent, 3 [7\%]).

Forty-nine patients (45\%) underwent cardiac surgery at the acute phase of IE. Among them, 29 (59\%) had CMBs, 20 (41\%) had acute ischemic lesions, and 15 (30.5\%) had both on MR imaging performed before surgery. None of these patients experienced clinically symptomatic cerebral complications after a mean follow-up of $20.3 \pm 19.4$ days.

In the present prospective study, we report a very high frequency of asymptomatic acute cerebral lesions detected in $71.5 \%$ of patients assessed by MR imaging. To the best of our knowledge, the present study is the largest prospective series reporting a comprehensive MR imaging description of asymptomatic cerebral lesions at the acute phase of IE and the first prospective one reporting CMBs in IE. The high prevalence of brain MR imaging lesions reflects both the poor sensitivity of neurologic examinations at the acute phase of IE and the embolic potential of valvular vegetation, larger than that attested to by clinical examination only.

To date, 2 previous prospective studies reported systematic MR imaging findings in patients with IE. In 1 study, the authors evaluated the incidence of cerebrovascular complications by systematic MR imaging in 60 patients with IE. ${ }^{11}$ They found that $65 \%$ of patients had cerebral lesions. Among them, $30 \%$ of patients had silent lesions at MR imaging. In another study of 40 patients, subclinical brain embolisms were reported in up to $48 \%$ of patients with left-sided IE. ${ }^{13}$ None of those studies reported CMBs or provided an extensive description of MR imaging findings. In the present study, CMBs were the most frequent cerebral abnormalities, in more than half of patients. Although none of the patients had undergone brain MR imaging before the onset of IE, different circumstances suggest a causal relationship between CMBs and IE. First, we reported, in a case-control study including part of the patients in our series, a specific association between CMBs and IE with an odds ratio of 10.06 (95\% confidence interval, 3.88-26.07). Moreover, the strength of the association between CMBs and IE increased with the number of CMBs, indicating a causal link. ${ }^{20}$ Furthermore, several other reports recently highlighted the occurrence of CMBs in IE. ${ }^{15,20-24}$

In IE, characteristics of CMBs seem slightly different compared with CMBs usually described in elderly patients and in cerebrovascular disease ${ }^{25-27}$; this difference may suggest varying un- 
derlying pathologic processes. In patients with IE, CMBs were mostly located in superficial areas and were obviously large and heterogeneous in signal. In a retrospective study of 11 patients, 7 had CMBs, with an increasing number of CMBs in 5 of them in subsequent MRI, suggesting an active process. ${ }^{21}$ In a patient in our series, a mycotic aneurysm confirmed angiographically was directly superimposed on a large and heterogeneous cortical $\mathrm{CMB}^{22}$ In addition, higher blood inflammatory marker levels (high-sensitivity C-reactive protein, interleukin 6 and 18) have been reported in patients with CMBs compared with those without them, independent of cardiovascular factors, raising the hypothesis that inflammation might be important in the pathogenesis of CMBs. ${ }^{28}$ Hence, CMBs in IE may result from a subacute inflammatory microvascular process. The occurrence of intracranial hemorrhage in IE has been recently associated with CMBs, suggesting a vascular vulnerability. ${ }^{23}$ In our series, we did not find an association between CMBs and mycotic aneurysms or parenchymal hemorrhage, which may be explained by the fact that our patients were asymptomatic, with few hemorrhagic lesions. In addition, anticoagulation therapy was reduced in patients with $>3 \mathrm{CMBs}$ undergoing anticoagulation; this result may partly explain a lack of symptomatic intracranial hemorrhage in our patients with CMBs who underwent cardiac surgery.

Acute ischemia was the second most frequent lesion, found in $36.7 \%$ of patients. Multiple watershed ischemic lesions were quite frequent, found in more than half of patients. Additionally, lesions of different ages with coexisting acute and subacute small infarcts were more numerous in those patients. Different patterns of ischemic lesions in IE visualized by using DWI have been previously reported in 36 infective and noninfective endocarditis cases with neurologic symptoms. ${ }^{29}$ In this retrospective study, larger ischemic lesions were more prevalent; this feature was consistent among a population of symptomatic patients.

In our study, CMBs and ischemia had different anatomic locations, which may support the different pathophysiology of these 2 types of lesions. The superficial (ie, cortical or meningeal) localization of CMBs in our patients may reflect a preferential mode of entry through the blood-brain barrier at the cortico-pial junction, whereas ischemic lesions result from an artery-to-artery embolic mechanism.

Cortical CMBs and multiple small watershed infarcts were the most frequent patterns observed in our large prospective series and could be used as a surrogate diagnostic marker in IE. Indeed, the diagnosis of IE remains difficult at the acute phase despite the use of the Duke criteria. ${ }^{16,30}$ Adding new imaging minor criteria may help in earlier diagnosis and appropriate therapeutic management. ${ }^{31}$ Our group recently reported that cerebral and abdominal imaging modified the diagnostic classification of IE and therapeutic plans. Duval et al ${ }^{15}$ showed that for 36 of 130 patients (28\%), the Duke classification for IE diagnosis and treatment options was modified on the basis of MR imaging findings. Iung et $\mathrm{al}^{14}$ found that both cerebral and abdominal MR imaging findings affected diagnosis but only cerebral MR imaging affected clinical management plans.
In clinical practice, unenhanced brain CT is mostly used for screening cerebral complications in IE because of its availability and rapidity. In case of suspected mycotic aneurysms, CT angiography has been reported to be comparable with 2D DSA for the detection of cerebral aneurysms. ${ }^{32}$ We chose MR imaging as the first-line imaging technique because of a better sensitivity in the detection of small parenchymal or meningeal complications and a high performance in the diagnosis of aneurysms. ${ }^{33}$ Additionally, MR imaging was preferentially used to avoid the substantial CT angiography contrast load, which can be of concern in patients with acute cardiac and/or renal failure in IE and/or in those receiving renal toxic drugs such as aminoglycosides.

Our study has several limitations. First, no follow-up imaging was systematically performed in our study. Therefore, the evolution of the detected lesions and the impact of cardiac surgery and anticoagulation of the extracorporeal circulation on their occurrence and/or evolution could not be assessed. Second, because no systematic conventional angiography was performed, some mycotic aneurysms could have been undiagnosed. However, minimally invasive techniques now have a more important role in the diagnosis of cerebral aneurysms. Indeed, MRA is highly accurate for the detection of cerebral aneurysms with the sensitivity of postcontrast MRA and 3D time-of-flight MR angiography reported to be, respectively, $95 \%-100 \%$ and $82 \%-96 \% .{ }^{33}$ Finally, some patients considered neurologically asymptomatic may have had subtle neurologic manifestations that could be masked by cardiovascular deficiency and septic syndrome in the context of acute IE. Indeed, in the study of Singhal et $\mathrm{al}^{29}$ of 36 patients with endocarditis with neurologic symptoms, $75 \%$ with small disseminated acute ischemic lesions on DWI had nonspecific encephalopathy symptoms. In our study, the absence of focal neurologic symptoms was verified by a standard neurologic examination (NIHSS), but without further analysis of neurocognitive functions.

\section{CONCLUSIONS}

Systematic MR imaging detects asymptomatic cerebral lesions in IE with a high prevalence. The radiologic description of cerebral lesions highlighted cortical CMBs and small watershed ischemic lesions as the most frequent pattern in this population. Further prospective studies are needed to evaluate the diagnostic accuracy of such MR imaging findings as surrogate criteria for IE diagnosis. Moreover, follow-up of patients is needed to assess the clinical relevance of brain MR imaging lesions to neurocognitive-function disorders, quality of life, and prognosis.

Disclosures: Agathe Hess—RELATED: Grant: Assistance Publique, Hôpitaux de Paris, Direction de la Recherche Clinique.* Bernard lung—RELATED: Grant: Project Hospitalier de Recherche Clinique, ${ }^{*}$ Comments: public funding, UNRELATED: Consultancy: Abbott, Bayer, Boehringer Ingelheim, Servier, Valtech, Payment for Lectures (including service on Speakers Bureaus): Edwards Lifesciences, Payment for Development of Educational Presentations: Sanofi Aventis, Travel/Accommodations/ Meeting Expenses Unrelated to Activities Listed: Abbott,* Boehringer Ingelheim.* Michel Wolff_-UNRELATED: Consultancy: Cubist Pharmaceutical, Trius Pharmaceutical, Hofmman Laroche, Comments: 1 meeting with each company in 2012, Travel/ Accommodations/Meeting Expenses Unrelated to Activities Listed: Astellas, Comments: payment for 2012 ICAAC (travel, accommodation, and inscription to the congress). Xavier Duval—RELATED: Grant: French Ministry of Health, ${ }^{*}$ UNRELATED: Travel/Accommodations/Meeting Expenses Unrelated to Activities Listed: Roche, ${ }^{*}$ GSK.* *Money paid to the institution.

AJNR Am J Neuroradiol 34:1579-84 Aug 2013 www.ajnr.org 


\section{REFERENCES}

1. Selton-Suty C, Célard M, Moing VL, et al. Preeminence of staphylococcus aureus in infective endocarditis: a 1-year population-based survey. Clin Infect Dis. 2012;54:1230-39

2. Heiro M, Nikoskelainen J, Engblom E, et al. Neurologic manifestations of infective endocarditis: a 17-year experience in a teaching hospital in Finland. Arch Intern Med 2000;160:2781-87

3. Pruitt AA, Rubin RH, Karchmer AW, et al. Neurologic complications of bacterial endocarditis. Medicine (Baltimore) 1978;57: $329-43$

4. Thuny F, Di Salvo G, Belliard O, et al. Risk of embolism and death in infective endocarditis: prognostic value of echocardiography: a prospective multicenter study. Circulation 2005;112:69-75

5. Chu VH, Cabell CH, Benjamin DK Jr, et al. Early predictors of inhospital death in infective endocarditis. Circulation 2004;109: 1745-49

6. Netzer RO, Zollinger E, Seiler C, et al. Infective endocarditis: clinical spectrum, presentation and outcome: an analysis of 212 cases 1980-1995. Heart 2000;84:25-30

7. Smith JM, So RR, Engel AM. Clinical predictors of mortality from infective endocarditis. Int J Surg 2007;5:31-34

8. Thuny F, Avierinos JF, Tribouilloy C, et al. Impact of cerebrovascular complications on mortality and neurologic outcome during infective endocarditis: a prospective multicentre study. Eur Heart $J$ 2007;28:1155-61

9. Moreillon P, Que YA. Infective endocarditis. Lancet 2004;363: $139-49$

10. Schonewille WJ, Singer MB, Atlas SW, et al. The prevalence of microhemorrhage on gradient-echo magnetic resonance imaging in acute lacunar infarction. J Stroke Cerebrovasc Dis 2005;14:141-44

11. Snygg-Martin U, Gustafsson L, Rosengren L, et al. Cerebrovascular complications in patients with left-sided infective endocarditis are common: a prospective study using magnetic resonance imaging and neurochemical brain damage markers. Clin Infect Dis 2008; 47:23-30

12. Takagi $Y$, Higuchi $Y$, Kondo $H$, et al. The importance of preoperative magnetic resonance imaging in valve surgery for active infective endocarditis. Gen Thorac Cardiovasc Surg 2011;59:467-71

13. Cooper HA, Thompson EC, Laureno R, et al. Subclinical brain embolization in left-sided infective endocarditis: results from the evaluation by MRI of the brains of patients with left-sided intracardiac solid masses (EMBOLISM) pilot study. Circulation 2009;120:585-91

14. Iung B, Klein I, Mourvillier B, et al. Respective effects of early cerebral and abdominal magnetic resonance imaging on clinical decisions in infective endocarditis. Eur Heart J Cardiovasc Imaging 2012;13:703-10

15. Duval X, Iung B, Klein I, et al. Effect of early cerebral magnetic resonance imaging on clinical decisions in infective endocarditis: a prospective study. Ann Intern Med 2010;152:497-504, W175

16. Durack DT, Lukes AS, Bright DK. New criteria for diagnosis of infective endocarditis: utilization of specific echocardiographic findings: Duke Endocarditis Service. Am J Med 1994;96:200-09

17. Chapot R, Houdart E, Saint-Maurice JP, et al. Endovascular treatment of cerebral mycotic aneurysms. Radiology 2002;222:389-96
18. Kannoth S, Thomas SV, Nair S, et al. Proposed diagnostic criteria for intracranial infectious aneurysms. J Neurol Neurosurg Psychiatry 2008;79:943-46

19. Fazekas F, Chawluk JB, Alavi A, et al. MR signal abnormalities at $\mathbf{1 . 5}$ $\mathrm{T}$ in Alzheimer's dementia and normal aging. AJR Am J Roentgenol 1987;149:351-56

20. Klein I, Iung B, Labreuche J, et al. Cerebral microbleeds are frequent in infective endocarditis: a case-control study. Stroke 2009;40: 3461-65

21. Morofuji Y, Morikawa M, Yohei T, et al. Significance of the T2*weighted gradient echo brain imaging in patients with infective endocarditis. Clin Neurol Neurosurg 2010;112:436-40

22. Klein I, Iung B, Wolff M, et al. Silent $T 2^{\star}$ cerebral microbleeds: a potential new imaging clue in infective endocarditis. Neurology 2007;68:2043

23. Okazaki S, Sakaguchi M, Hyun B, et al. Cerebral microbleeds predict impending intracranial hemorrhage in infective endocarditis. Cerebrovasc Dis 2011;32:483-88

24. Subramaniam S, Puetz V, Dzialowski I, et al. Cerebral microhemorrhages in a patient with mycotic aneurysm: relevance of T2-GRE imaging in SBE. Neurology 2006;67:1697

25. Cordonnier C, Al-Shahi Salman R, Wardlaw J. Spontaneous brain microbleeds: systematic review, subgroup analyses and standards for study design and reporting. Brain 2007;130:1988-2003

26. Fazekas F, Kleinert R, Roob G, et al. Histopathologic analysis of foci of signal loss on gradient-echo $\mathrm{T} 2^{\star}$-weighted MR images in patients with spontaneous intracerebral hemorrhage: evidence of microangiopathy-related microbleeds. AJNR Am J Neuroradiol 1999;20: $637-42$

27. Viswanathan A, Chabriat H. Cerebral microhemorrhage. Stroke 2006;37:550-55

28. Miwa K, Tanaka M, Okazaki S, et al. Relations of blood inflammatory marker levels with cerebral microbleeds. Stroke 2011;42: 3202-06

29. Singhal AB, Topcuoglu MA, Buonanno FS. Acute ischemic stroke patterns in infective and nonbacterial thrombotic endocarditis: a diffusion-weighted magnetic resonance imaging study. Stroke 2002;33:1267-73

30. Li JS, Sexton DJ, Mick N, et al. Proposed modifications to the Duke criteria for the diagnosis of infective endocarditis. Clin Infect Dis 2000;30:633-38

31. Dickerman SA, Abrutyn E, Barsic B, et al. The relationship between the initiation of antimicrobial therapy and the incidence of stroke in infective endocarditis: an analysis from the ICE Prospective Cohort Study (ICE-PCS). Am Heart J 2007; 154:1086-94

32. Yoon DY, Lim KJ, Choi CS, et al. Detection and characterization of intracranial aneurysms with 16-channel multidetector row CT angiography: a prospective comparison of volume-rendered images and digital subtraction angiography. AJNR Am J Neuroradiol 2007;28:60-67

33. Unlu E, Cakir B, Gocer B, et al. The role of contrast-enhanced MR angiography in the assessment of recently ruptured intracranial aneurysms: a comparative study. Neuroradiology 2005;47:780-91 